\title{
Searches for Higgs bosons beyond the standard model at the Tevatron
}

\author{
Christopher Hays* \\ Oxford University \\ E-mail: hays@physics.ox.ac.uk
}

\begin{abstract}
There are many reasons to expect new non-standard-model processes at the electroweak scale, and additional Higgs bosons are a common feature of new-physics models. Recent Tevatron searches have expanded the sensitivity to Higgs bosons predicted by numerous models, including the minimal and next-to-minimal supersymmetric models.
\end{abstract}

XVIII International Workshop on Deep-Inelastic Scattering and Related Subjects, DIS 2010 April 19-23, 2010

Firenze, Italy

${ }^{*}$ Speaker. 


\section{Introduction}

There are a variety of large-scale phenomena, such as the existence of dark matter, dark energy, and the matter-antimatter asymmetry, that cannot be explained by the standard model of particle physics (SM). Many new-physics models have been proposed to provide fundamental sources of these phenomena, and these frequently require additional Higgs bosons to break a symmetry in the original Lagrangian or to maintain consistency with other established phenomena. For example, supersymmetry provides a dark matter candidate and requires a second Higgs SU(2) doublet beyond that of the SM. While new Higgs bosons could have masses too large to be accessible at current colliders, there are good reasons to expect at least some new Higgs bosons at the electroweak mass scale. In particular, the current density of dark matter requires the cross section of dark matter annihilation to be of the order of a picobarn, similar to typical electroweak processes. It is thus natural for the dark matter particle to have a mass of $\mathscr{O}\left(100 \mathrm{GeV} / \mathrm{c}^{2}\right)$ and a connection to electroweak symmetry breaking. This expectation of non-SM physics at the electroweak mass scale makes searches for new Higgs bosons a promising avenue for discovery at the Tevatron collider.

Higgs bosons beyond the SM typically share the mass-dependent-coupling feature of the SM Higgs boson. However, different SM particles can get their masses from different Higgs bosons, affecting Higgs boson phenomenology. In addition, the Higgs boson(s) may decay to new non-SM particles, allowing many existing limits to be partially or completely evaded. Dedicated searches at the Tevatron for Higgs bosons within the minimal and next-to-minimal supersymmetric models have significantly expanded sensitivity to new Higgs bosons with a wide variety of properties.

\section{Higgs bosons in minimal supersymmetry}

In the minimal supersymmetric model (MSSM) the Higgs supermultiplet is expanded to contain eight degrees of freedom: the longitudinal components of the $W$ and $Z$ bosons $\left(W_{L}^{ \pm}, Z_{L}^{0}\right)$, two neutral CP-even Higgs bosons $\left(h^{0}\right.$ and $H^{0}$, where $\left.m_{h^{0}}<m_{H^{0}}\right)$, one neutral CP-odd Higgs boson $\left(A^{0}\right)$, and one charged Higgs boson $\left(H^{ \pm}\right)$. Two Higgs $\mathrm{SU}(2)$ doublets, $H_{u}$ and $H_{d}$, are required for these eight degrees of freedom, and their vacuum expectation values give the SM fermions and gauge bosons their masses (e.g., $m_{u}=\lambda_{u} v_{u}$ and $m_{d}=-\lambda_{d} v_{d}$ ). The physical Higgs bosons are rotations of $H_{u}$ and $H_{d}$; the neutral fields can be expressed as

$$
\left(\begin{array}{c}
H_{u}^{0} \\
H_{d}^{0}
\end{array}\right)=\left(\begin{array}{c}
v_{u} \\
v_{d}
\end{array}\right)+\frac{1}{\sqrt{2}} R_{\alpha}\left(\begin{array}{c}
h^{0} \\
H^{0}
\end{array}\right)+\frac{i}{\sqrt{2}} R_{\beta}\left(\begin{array}{c}
Z_{L}^{0} \\
A^{0}
\end{array}\right),
$$

where $R_{\alpha}$ and $R_{\beta}$ are usual rotation matrices. The angle $\beta$ is related to the Higgs vacuum expectation values through $\tan \beta=v_{u} / v_{d}$. Searches at the Large Electron-Positron collider (LEP) generally place a lower limit on $\tan \beta$ of $\mathscr{O}(1)$. The $W$ and $Z$ boson masses fix the quadrature sum of $v_{u}$ and $v_{d}$ and suggest $\lambda_{t} \sim 1$ for $\tan \beta>1$. If $\lambda_{b}$ also has a natural value of about 1 , then $\tan \beta \sim 40$, motivating searches at large $\tan \beta$.

In this regime, the neutral Higgs bosons and enhanced couplings to down-type fermions (e.g., $\lambda_{t} \sim \lambda_{t}^{S M} \cot \beta$ and $\lambda_{b} \sim \lambda_{b}^{S M} \tan \beta$ ) and $A^{0}$ has a mass similar to $h^{0}$ or $H^{0}$. The enhanced couplings result in a significant production cross section for the process $b \bar{b} \rightarrow h / H / A$ [1], and the approximate mass degeneracy effectively doubles the cross section. The Higgs bosons decay predominantly to $b \bar{b}$ and $\tau \bar{\tau}$, so the Tevatron search signature is a pair of these particles and up to two $b$ quarks. 

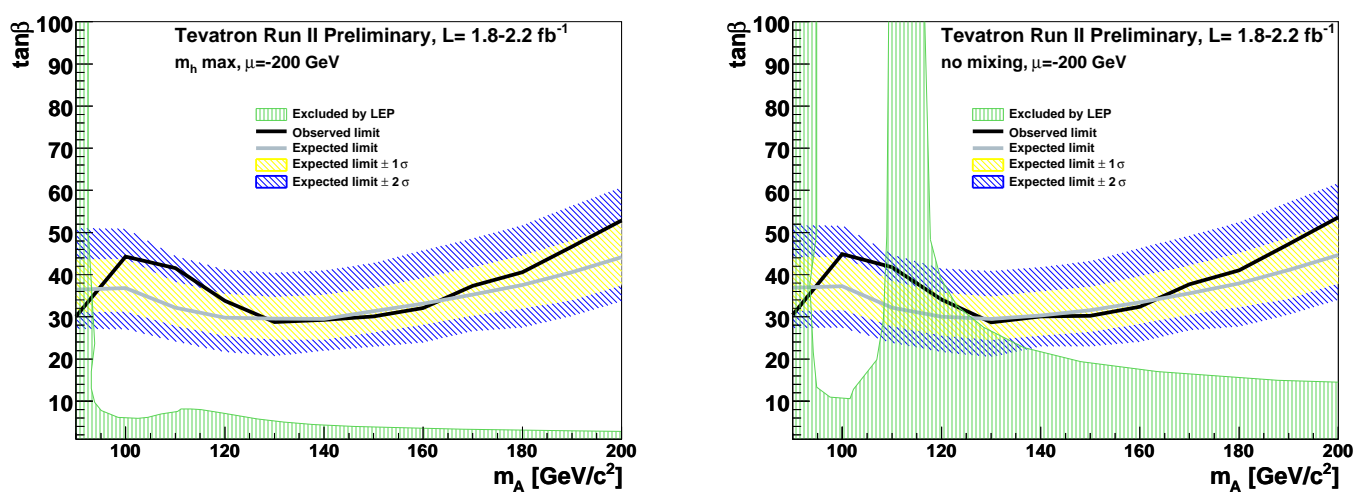

Figure 1: The Tevatron combined exclusion in the $m_{A}-\tan \beta$ plane for two MSSM scenarios (" $m_{h}$ max" (left) and "no mixing" (right) [1]). The line of 95\% C.L. exclusion is shown in black, and the region above the line is excluded at $>95 \%$ C.L.

\subsection{Searches for Higgs boson decays to tau leptons}

The CDF and DØ collaborations have searched for $H / A \rightarrow \tau \tau$ with and without requiring an associated $b$-quark. The inclusive searches cover the final states where one or both tau leptons decay to an electron or muon. Potential Higgs boson signal events are distinguished from the large Drell-Yan background using the "visible" mass, defined as the invariant mass of the hadrons and charged leptons from the tau decays and the transverse momentum imbalance of the event).

No significant signal is seen in the inclusive CDF search based on $1.8 \mathrm{fb}^{-1}$ [2], or the inclusive $\mathrm{D} \emptyset$ search based on either $1 \mathrm{fb}^{-1}$ [3] or $2.2 \mathrm{fb}^{-1}$. The collaborations have combined their individual results [4] to produce a 95\% confidence level (C.L.) exclusion curve in the $m_{A}-\tan \beta$ plane (Fig. 1). For $\tan \beta=40$, masses between $\approx 115-170 \mathrm{GeV} / c^{2}$ are excluded in particular MSSM scenarios.

The $\mathrm{D} \varnothing$ collaboration has additionally searched $2.7 \mathrm{fb}^{-1}$ of data for $H / A \rightarrow \tau \tau$ produced with at least one $b$-quark [5]. The $b$-quark requirement suppresses Drell-Yan production by nearly two orders of magnitude and improves sensitivity near the $Z$ boson mass peak. The leading residual backgrounds are $t \bar{t}$ and multijet production, and are respectively reduced using a neural network (Fig. 2) and a likelihood. A small excess is observed in data relative to background expectation (79 events observed compared to $68.7 \pm 2.0$ expected), making the observed limits in the $m_{A}-\tan \beta$ plane (Fig. 2) less stringent than those obtained from the combined limits in the inclusive search.

\subsection{Searches for Higgs boson decays to $b$-quarks}

Searches for Higgs bosons decaying to $b$-quarks are challenging because of larger and more complex combinations of backgrounds. $\mathrm{CDF}$ and $\mathrm{D} \emptyset$ select events with at least three identified $b$-quark jets and produce mass templates of the QCD background production of three $b$-quarks or a mix of $b$-quarks and light quarks or gluons. To suppress background D $\emptyset$ uses a likelihood of six variables [6], while CDF directly fits the mass distribution of the two jets with the highest $E_{T}$. In $2.5 \mathrm{fb}^{-1}$ of data, CDF observes a small excess for $m_{H}$ roughly equal to $150 \mathrm{GeV} / c^{2}$; however, $\mathrm{\varnothing}$ see no such excess in $2.6 \mathrm{fb}^{-1}$ of data. Limits in the $m_{A}-\tan \beta$ plane are not as stringent as in the tau lepton search, excluding $\tan \beta=40$ only for $m_{A} \approx 120 \mathrm{GeV} / c^{2}$ (DØ) or $90 \mathrm{GeV} / c^{2}$ (CDF). 

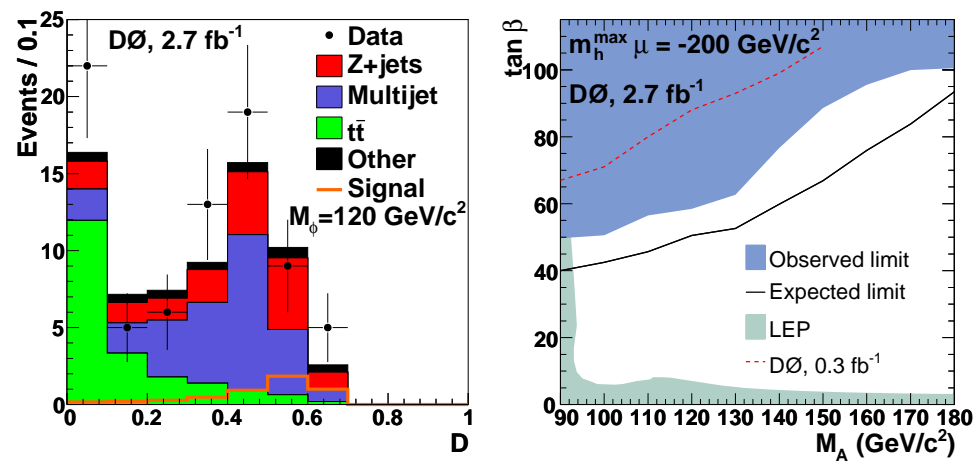

Figure 2: Left: The neural network discriminant used to separate $t \bar{t}$ production from Higgs boson signal in the $\mathrm{D} \varnothing$ search for $H \rightarrow \tau \bar{\tau}$ produced in association with at least one $b$-quark. Right: The blue-shaded region excluded at $\geq 95 \%$ C.L. in the $m_{A}-\tan \beta$ plane for the " $m_{h}$ max" scenario.
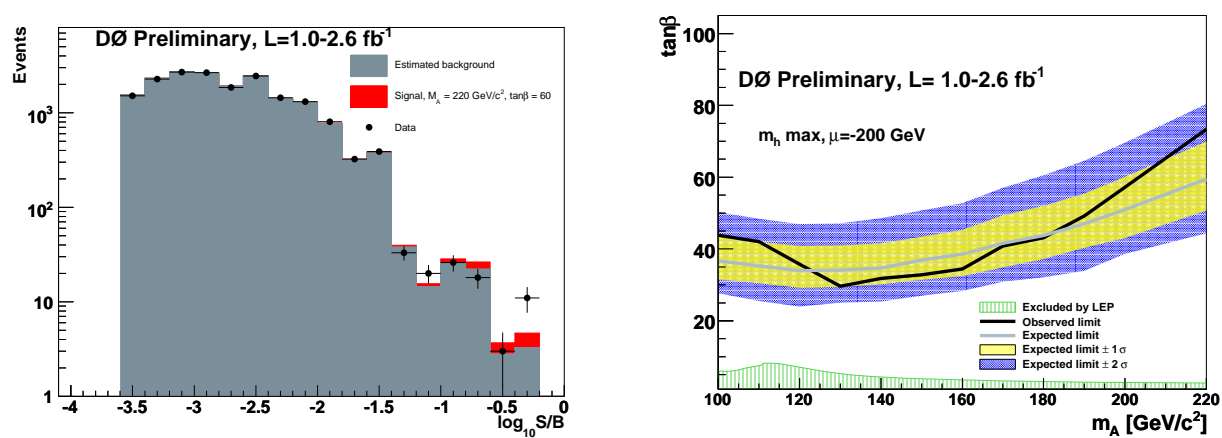

Figure 3: Left: The events expected and observed by the combined D $\varnothing$ MSSM Higgs boson searches at $m_{H}=220 \mathrm{GeV} / c^{2}$, as a function of the logarithm of signal over background. A small excess is observed near $\mathrm{S} / \mathrm{B}=1$. Right: The 95\% C.L. exclusion line (black) in the $m_{A}-\tan \beta$ plane.

\subsection{Combination of MSSM searches}

$\mathrm{D} \varnothing$ has combined its searches in the $\tau \tau, \tau \tau b, b b b$ and $b b b b$ final states, covering 19 channels defined by tau decay mode and jet multiplicity. The limits in the $m_{A}-\tan \beta$ mass plane (Fig. 3) are similar to those from the $\mathrm{CDF}$ and $\mathrm{D} \varnothing$ combined limits from the searches for $H / A \rightarrow \tau \bar{\tau}$. The combined data are in good agreement with the SM prediction.

\section{Higgs bosons in next-to-minimal supersymmetry}

One attractive feature of supersymmetry is that it solves a naturalness problem, namely that loop corrections to the Higgs boson mass tend to give it a large mass of order the cutoff scale (expected to be $\approx M_{P l}$ ). Loops with supersymmetric (scalar) particles have opposite sign to fermionic loops, resulting in a destructive interference that maintains a Higgs boson mass of order the supersymmetry breaking scale. One problem with this picture is that there is an additional Higgs boson mass term in the supersymmetric Lagrangian, the so-called " $\mu$ " term, that should also be 
$\approx M_{P l}$. This can be solved by invoking a symmetry that eliminates this term from the fundamental Lagrangrian, and instead produce it via a symmetry-breaking mechanism at the electroweak scale.

Such a mechanism occurs in the next-to-minimal supersymmetric model (NMSSM), which has an SM-singlet scalar field $S$ and a term in the superpotental $\left(\lambda_{S} S H_{d} H_{u}\right)$ that can produce a $\mu$ term $\left(=\lambda_{S}<S>\right.$ ). The new field results in two Higgs bosons, $a^{0}$ (CP-odd) and $h_{S}^{0}$ (CP-even), that are potential decay modes for $h^{0}$. In fact, the branching ratio of $h^{0}$ to SM particles can be as low as a few percent for $m_{h^{0}} \lesssim 140 \mathrm{GeV} / c^{2}$. In this scenario, the LEP limits on the SM Higgs boson can be evaded and $m_{h^{0}}$ could be $\mathscr{O}(100) \mathrm{GeV} / c^{2}$. A recent search from LEP [7] has narrowed the allowed parameter space, but the Tevatron is expected to have significant additional sensitivity.

DØ has performed the first search for $h^{0}$ produced via gluon fusion and decaying to $a^{0}$ pairs [8]. To suppress background, D $\varnothing$ requires one $a^{0}$ to decay to $\mu \bar{\mu}$. A small excess of 4 observed events compared to $1.1 \pm 0.2$ background appears for $m_{a^{0}}=4 \mathrm{GeV} / c^{2}$, but the rate is well above the expectation for gluon fusion at $m_{h}^{0}=100 \mathrm{GeV} / c^{2}$. For $2 m_{\mu}<a_{0}<2 m_{\tau}$, D $\varnothing$ constrains the branching ratio for $a_{0}$ to muon pairs to be less than $7 \%$, restricting the NMSSM parameter space.

CDF has searched for $a_{0}$ in the decay $t \rightarrow H^{+} \rightarrow W^{+} a_{0}$ in a scenario where $a_{0} \rightarrow \tau \bar{\tau}$. This scenario could explain the $2.8 \sigma$ deviation at LEP in $W \rightarrow \tau v / W \rightarrow l v$. CDF selects events with a lepton and 3 hadronic jets (one of which is tagged to originate from a $b$-quark). To separate signal from the large SM $t \bar{t}$ background, an additional isolated track is required, reducing background by $90 \%(835 \rightarrow 83)$. No excess is observed in the distribution of isolated track $p_{T}$, and upper bounds of $\mathscr{O}(10 \%)$ are set on $\mathrm{BR}\left(t \rightarrow H^{+} b\right)$ if $m_{H^{+}} \lesssim 135 \mathrm{GeV} / c^{2}$ and $\mathrm{BR}\left(H^{ \pm} \rightarrow W^{ \pm} A\right)=1$.

\section{Summary}

Tevatron searches for Higgs bosons beyond the SM probe interesting regions of supersymmetric parameter space motivated both by naturalness considerations and by gaps in LEP coverage. Other searches include "fermiophobic" Higgs bosons coupling only to other bosons, where the Tevatron limits [9] are now similar to those from LEP, and doubly charged Higgs bosons predicted by see-saw models explaining low neutrino masses. With a factor of 2-4 more data available for analysis, the Tevatron will continue to provide new sensitivity to non-SM Higgs bosons.

\section{References}

[1] M. Carena, S. Heinemeyer, C.E.M. Wagner, and G. Weiglein, Eur. Phys. J. C 45, 797 (2006).

[2] T. Aaltonen et al. (CDF Collaboration), Phys. Rev. Lett. 103, 201801 (2009).

[3] V. M. Abazov et al. (DØ Collaboration), Phys. Rev. Lett. 101, 071804 (2008).

[4] CDF and DØ Collaborations and Tevatron New Physics Higgs WG, arXiv:1003.3363v3 (2010).

[5] V. M. Abazov et al. (DØ Collaboration), Phys. Rev. Lett. 104, 151801 (2010).

[6] V. M. Abazov et al. (DØ Collaboration), Phys. Rev. Lett. 101, 221802 (2008).

[7] S. Schael et al. (ALEPH Collaboration), J. High En. Phys. 05, 049 (2010).

[8] V. M. Abazov et al. (DØ Collaboration), Phys. Rev. Lett. 103, 061801 (2009).

[9] T. Aaltonen et al. (CDF Collaboration), Phys. Rev. Lett. 103, 061803 (2009); V. M. Abazov et al. (DØ Collaboration), Phys. Rev. Lett. 101, 051801 (2008). 